PROCEEDINGS OF THE

AMERICAN MATHEMATICAL SOCIETY

Volume 126, Number 9, September 1998, Pages 2593-2597

S $0002-9939(98) 04386-\mathrm{X}$

\title{
A TOPOLOGY ON LATTICE ORDERED GROUPS
}

\author{
IVICA GUSIĆ \\ (Communicated by Roe Goodman)
}

\begin{abstract}
We show that a lattice ordered group can be topologized in a natural way. The topology depends on the choice of a set $C$ of admissible elements ( $C$-topology). If a lattice ordered group is 2-divisible and satisfies a version of Archimedes' axiom ( $C$-group), then we show that the $C$-topology is Hausdorff. Moreover, we show that a $C$-group with the $C$-topology is a topological group.
\end{abstract}

In section 1 we recall the definition as well as the elementary properties of lattice ordered groups, especially the properties of the norm $N$ on such groups.

In section 2 we introduce the notion of a set of admissible elements $C$ (definition 2 ) in a lattice ordered 2-divisible group $A$. It is proved (lemma 2) that the sets $U_{x_{0}, r}=\left\{x \in A: r-N\left(x-x_{0}\right) \in C\right\}$ constitute a base of a topology on $A$ (the $C$-topology).

In section 3 we describe the properties of the $C$-topology on lattice ordered 2divisible $C$-Archimedean groups (such groups are called $C$-groups). It is proved that $C$-groups are Hausdorff topological groups (theorem 1 and theorem 2).

\section{LATTICE ORDERED GROUPS}

A lattice ordered group [1, Ch. VI, $\S \S 8,9]$ is an ordered group $A$ such that there exist $\sup (x, y)$ and $\inf (x, y)$, for every $x, y \in A$. Note that:

$$
\inf (x, y)=-\sup (-x,-y)
$$

Definition 1 ([1, VI, definition 4]). The norm $N$ on a lattice ordered group $A$ is the function $N: A \rightarrow A$ defined by $N(x)=\sup (x,-x)$.

Lemma 1. Let $N$ be the norm on a lattice ordered group $A$. Then:

(i) $N(x)=\sup (x, 0)-\inf (x, 0)$ for every $x, y \in A$.

(ii) $N(x)=x$ if and only if $x \geq 0$. In particular, $N(N(x))=N(x)$, for every $x \in A$.

(iii) $N(x) \geq 0$, for every $x \in A$.

(iv) $(N(x)=0) \Leftrightarrow(x=0)$, for every $x \in A$.

(v) $N(m x)=|m| N(x)$, for every $x \in A$ and for every $m \in \mathbf{Z}$.

(vi) $N(x+y) \leq N(x)+N(y)$, for all $x, y \in A$.

Proof. See [1, VI, Proposition 9 and corollary 4 of Proposition 11].

Received by the editors February 28, 1996 and, in revised form, February 13, 1997.

1991 Mathematics Subject Classification. Primary 06F30, 22A99.

Key words and phrases. Lattice ordered group, set of admissible elements, $C$-topology, $C$ group.

(C) 1998 American Mathematical Society 
Directly from definition 1 we conclude that:

$$
N(x) \leq \epsilon \Leftrightarrow-\epsilon \leq x \leq \epsilon
$$

for all $\epsilon \geq 0, x \in A$. In particular, $-N(x) \leq x \leq N(x)$, for every $x \in A$.

\section{The $C$-Topology}

In this section we suppose that $A$ is a lattice ordered 2-divisible group. Note that in a lattice ordered group there is no nontrivial torsion element. Namely, $n x=0$, for $n \in \mathbf{N}$, implies $n N(x)=0$ or equivalently $(n-1) N(x)=-N(x)$; hence $N(x)=0$, and so $x=0$ (lemma 1 (iii), (iv) and (v)). Therefore, $\frac{x}{2}$ is uniquely determined, for every $x \in A$. It is easily seen (by lemma 1 , (ii) and (v)) that $x \geq 0$ implies $\frac{x}{2} \geq 0$.

Definition 2. A set of admissible elements in a lattice ordered 2-divisible group $A$ is any nonempty subset $C$ of the set $A^{+}$of all positive elements having the following properties:

(i) $0 \notin C$,

(ii) $(x \in C \wedge y \geq x) \Rightarrow(y \in C)$,

(iii) $(x, y \in C) \Rightarrow(\inf (x, y) \in C)$,

(iv) $(x \in C) \Rightarrow\left(\frac{x}{2} \in C\right)$.

It is obvious that $C \subseteq A^{+} \backslash\{0\}$. If there exist at least two coprime elements in the group, then the given inclusion is strict (by (iii), (i) of definition 2 and the fact that coprime elements are necessarily positive). Recall that $x, y \in A$ are coprime if $\inf (x, y)=0([1, \mathrm{~V}$, definition 5$])$.

Remark. Suppose that $a$ is a strictly positive element of $A$. Put $A_{a}=\{x \in A$ : $x \geq a\}$. Then $A_{a}$ satisfies (i), (ii), (iii) of definition 2. Denote $A_{a, n}=\frac{1}{2^{n-1}} A_{a}$, for $n \in \mathbf{N}$. Then we have $A_{a, n+1} \supseteq A_{a, n}$, for every $n \in \mathbf{N}$. Put $C=\bigcup_{n \in \mathbf{N}} A_{a, n}$. Then $C$ is a set of admissible elements. This is the minimal set of admissible elements containing $a$.

Recall that the open ball in a normed space is defined by the relation $r-\left|x-x_{0}\right|>$ 0 . In our case we have

Definition 3. Let $A$ be a lattice ordered 2-divisible group and let $C$ be a set of admissible elements of $A$. The open $C$-ball of radius $r \in C$, with the centre $x_{0} \in A$, is the set of all $x \in A$ such that $r-N\left(x-x_{0}\right) \in C$. We denote this set by $U_{x_{0}, r}$.

Lemma 2. Let $A$ be a lattice ordered 2-divisible group. Then open $C$-balls constitute a base of a topology on $A$ (we called this topology the $C$-topology).

Proof. Since $x_{0} \in U_{x_{0}, r}$, for all $r \in C$, we get that open $C$-balls form an open cover of the space $A$. Let $z_{0} \in U_{x_{0}, r} \cap U_{y_{0}, R}$ be arbitrary. By the definition of $C$-balls, we have $r-N\left(z_{0}-x_{0}\right)=c_{1}$ and $R-N\left(z_{0}-y_{0}\right)=c_{2}$, for some $c_{1}, c_{2} \in C$. Then $U_{x_{0}, \epsilon} \subseteq U_{x_{0}, r} \cap U_{y_{0}, R}$, for $\epsilon=\inf \left(c_{1}, c_{2}\right)$. Namely, for $x \in U_{x_{0}, r}$, we have $\epsilon-N\left(x-x_{0}\right)=c_{3}$, for some $c_{3} \in C$. Applying the triangle inequality (lemma 1 , (vi)), we get $r-N\left(x-x_{0}\right) \geq r-N\left(x_{0}-z_{0}\right)+\epsilon-N\left(z_{0}-x\right)-\epsilon=c_{1}+c_{3}-\epsilon \in C$. Similar reasoning holds for the second ball. This completes the proof.

Remark. Lemma 2 is valid even if $A$ is not necessarily 2-divisible. Hence $C$ does not need to satisfy (iv) of definition 2 .

It is easy to see that if $V_{x_{0}, r}=\left\{x \in A: N\left(x-x_{0}\right)<r\right\}$ and $F_{x_{0}, r}=\{x \in A$ : $\left.N\left(x-x_{0}\right) \leq r\right\}$, then we have $U_{x_{0}, r} \subseteq V_{x_{0}, r} \subseteq F_{x_{0}, r}$. 
Example 1. Let $A=\mathbf{R}^{2}$ with the relation of order defined by $(a, b) \leq(c, d) \Leftrightarrow$ $(a \leq c \wedge b \leq d)$. Then $A$ is a divisible lattice ordered group such that

$$
\sup ((a, b),(c, d))=(\sup (a, c), \sup (b, d)), \quad A^{+}=\{(a, b): a \geq 0 \wedge b \geq 0\},
$$

and $C=\{(a, b): a>0 \wedge b>0\}$ is the set of admissible elements. It is easy to see that:

$U_{\left(x_{0}, y_{0}\right), r}=\left\{(x, y) \in \mathbf{R}^{2}:-r<x-x_{0}<r \wedge-r<y-y_{0}<r\right\} ;$

$F_{\left(x_{0}, y_{0}\right), r}=\left\{(x, y) \in \mathbf{R}^{2}:-r \leq x-x_{0} \leq r \wedge-r \leq y-y_{0} \leq r\right\}$;

$V_{\left(x_{0}, y_{0}\right), r}=F_{\left(x_{0}, y_{0}\right), r} \backslash\left\{\left(x_{0}+r, y_{0}+r\right),\left(x_{0}+r, y_{0}-r\right),\left(x_{0}-r, y_{0}+r\right),\left(x_{0}-r, y_{0}-r\right)\right\}$.

Thus, the $C$-topology on $A$ is equivalent to the standard topology on $\mathbf{R}^{2}$.

It can be shown that the set $\left\{(x, y) \in \mathbf{R}^{2}:(x \geq 0) \wedge(y>0)\right\}$ is a set of admissible elements, too. Of course, in this case the corresponding $C$-topology is not equivalent to the previous one.

Note that the norm $N$ is a continuous function with respect to the $C$-topology. Namely, by the triangle inequality, we have $-N(x-y) \leq N x-N y \leq N(x-y)$, so by $(2), N(N x-N y) \leq N(x-y)$.

Lemma 3. Let $A$ be a lattice ordered 2-divisible group, and let $C$ be a set of admissible elements. Then:

(i) $C$ is an open set in the $C$-topology.

(ii) $A=C-C$.

Proof. (i) The inequality $N(x-c) \leq \frac{c}{2}$ is, by (2), equivalent to the inequalities $\frac{c}{2} \leq x \leq \frac{3 c}{2}$, for every $c \in C$. Hence, $U_{c, \frac{c}{2}} \subseteq C$.

(ii) By lemma 1, (i) and (1), we have

$$
x=\sup (x, 0)-\sup (-x, 0)=(\sup (x, 0)+c)-(\sup (-x, 0)+c)
$$

for all $x \in A, c \in C$.

\section{3. $C$-groups}

In this section we will assume that $A$ is a lattice ordered 2-divisible $C$-Archimedean group. This means that in the group $A$ the following version of Archimedes' axiom holds:

$$
(\forall x \in C)(\forall y \in C)(\exists n \in \mathbf{N})(n \cdot y>x) .
$$

One can see that (3) is equivalent to

$$
(\forall x \geq 0)(\forall y \in C)(\exists n \in \mathbf{N})(n \cdot y>x) .
$$

A consequence of the given assumption is that $U \cap C \neq \emptyset$, for every neighbourhood $U$ of zero. Namely, if $U$ is the open $C$-disc around zero of radius $y$ and if $x \in C$ is arbitrary, then there exists $n \in \mathbf{N}$ such that $y-\frac{x}{2^{n}} \in C$. Hence $\frac{x}{2^{n}} \in U \cap C$.

Definition 4. We say that $A$ is a $C$-group if $A$ is a lattice ordered, 2-divisible, $C$-Archimedean group.

Example 2. Let $A$ be $\mathbf{R}^{2}$ as in example 1. If we choose $C=\{(x, y): x>0, y>0\}$, then $A$ becomes a $C$-group. If we choose $C=\{(x, y): x \geq 0, y>0\}$, then $A$ is not a $C$ - group. 
Example 3. The group $\mathbf{Q}_{2}$ of all dyadic numbers, with the standard ordering, is a $C$-group ( $C$ is the set of strictly positive dyadic numbers). The closure $\mathrm{ClQ}_{2}$ is the additive group of real numbers. Note that every $C$-group is a module over the dyadic numbers.

Lemma 4. Let $A$ be a $C$-group. Then for all $x \in A$ and $c \in C$ there exists $n \in \mathbf{N}$ such that $\frac{x}{2^{n}}+c \in C$.

Proof. Let $c \in C, x \in A$. Then, by lemma 3 (ii), there exist $c_{1}, c_{2} \in C$ such that $x=c_{1}-c_{2}$. Hence, $\frac{x}{2^{n}}=\frac{c_{1}}{2^{n}}-\frac{c_{2}}{2^{n}}$, for every $n \in \mathbf{N}$. If we choose $n$ such that $c-\frac{c_{2}}{2^{n}}>0$ (this is possible because the group $A$ is $C$-Archimedean), then we have $\frac{x}{2^{n}}+c=\frac{c_{1}}{2^{n}}+\left(c-\frac{c_{2}}{2^{n}}\right) \in C$.

Recall that, by the definition, $x \in A$ is a limit of the sequence $\left(x_{n}\right)$ if the following holds: $(\forall \epsilon \in C)\left(\exists n_{0} \in \mathbf{N}\right)\left(\forall n \geq n_{0}\right)\left(\epsilon-N\left(x-x_{n}\right) \in C\right)$.

Lemma 5. (i) Let $F \subseteq A$. Then the closure of $F$ is $\mathrm{Cl} F=\left\{\lim x_{n}: x_{n} \in F\right\}$.

(ii) $A^{+}=\mathrm{ClC}$.

Proof. (i) Let the set of all limits of the sequences with terms from $F$ be denoted by $X$.

Suppose that $x \in X$. Then every open neighbourhood of $x$ cuts $F$. Therefore, $X \subseteq \mathrm{Cl} F$. Suppose that $x \in \mathrm{Cl} F$ and that $\epsilon \in C$. Let $U_{n}$ be an open $C$-disc around $x$ with radius $\frac{\epsilon}{2^{n}}$. By definition, $U_{n} \cap F \neq \emptyset$, for every $n \in \mathbf{N}$. Choose $x_{n} \in U_{n} \cap F$, for every $n \in \mathbf{N}$. Then $x=\lim x_{n}$. Therefore $\mathrm{Cl} F \subseteq X$. (ii) Suppose that $x \in A^{+}$. Choose an arbitrary $c \in C$. Then $\lim \left(x+\frac{c}{2^{n}}\right)=x$ and $x+\frac{c}{2^{n}} \in C$ for all $n \in \mathbf{N}$. Hence, $x \in \mathrm{ClC}$.

Suppose that $x \in \mathrm{ClC}$. Then $x=\lim x_{n}$, for $x_{n} \in C$. Applying the continuity of the norm $N$ and lemma 1 (ii), we get $N x=N\left(\lim x_{n}\right)=\lim \left(N x_{n}\right)=\lim x_{n}=x$. Hence, $x \geq 0$.

Lemma 6. Suppose that $x \geq 0$ and that $x<c$, for every $c \in C$. Then $x=0$.

Proof. Let $x=\lim x_{n}, x_{n} \in C$ for all $n \in \mathbf{N}$ (this is possible by lemma 5, (ii)). Therefore, $N\left(x-x_{n}\right)<\epsilon$, for every sufficiently large $n \in \mathbf{N}$, and for arbitrary $\epsilon \in C$. Hence, $-\epsilon<x-x_{n}<\epsilon$, and so, $x_{n}<x+\epsilon<2 \epsilon$, for every sufficiently large $n \in \mathbf{N}$. Thus, $\left(x_{n}\right)$ converges to zero; hence, $x=0$.

Theorem 1. A is a Hausdorff space.

Proof. Suppose that $x, y \in A$ and $x \neq y$. If $N(x-y)=\epsilon \in C$, then $\frac{\epsilon}{4}$ neighbourhoods around $x$ and around $y$ are disjoint. Suppose that $N(x-y) \in$ $A^{+} \backslash C$. After a translation we can assume that $y=0$ and $N x \in A^{+} \backslash C$ (note that $N x \neq 0$ ). If we show that 0 and $N x$ can be separated by open $C$-balls, then we can conclude that 0 and $x$ can be separated, too. Namely, if $U$ is an open $C$-ball around 0 and $W$ an open $C$-ball around $N x$ which are disjoint, then $U$ is disjoint from an open $C$-ball $V$ around $x$ such that $N V \subset W$ ( $V$ exists because $N$ is a continuous function). If not, there exists $z \in U \cap V$. Then we get $N z \in N(U \cap V) \subseteq N U \cap N V \subseteq U \cap V$, a contradiction (note that by the definition of $C$-balls we have $N U \subset U)$.

Therefore, we can suppose that $x \in A^{+} \backslash C$ and $x \neq 0$. It can be easily seen that 0 can be separated from $x$. If not, we get that $x \in U$, for every open neighbourhood $U$ around zero. Applying lemma 6 , we conclude that $x=0$ (a contradiction). Let's 
prove that $x$ can be separated from 0 . Choose $U=U_{\epsilon}$ an open $C$-ball of radius $\epsilon$ around zero, $\epsilon \in C$, such that $x \notin U_{2 \epsilon}$. Then $x \in V=U_{x+\frac{\epsilon}{2}, \epsilon}$. We claim that $U \cap V=\emptyset$. If not, there exists $z \in U \cap V$; hence, $N z<\epsilon$ and $N\left(z-x-\frac{\epsilon}{2}\right)<\epsilon$. Therefore, $x+\frac{\epsilon}{2}=N\left(x+\frac{\epsilon}{2}\right) \leq N\left(x+\frac{\epsilon}{2}-z\right)+N z<2 \epsilon$; hence, $x<\frac{3 \epsilon}{2}$. This contradicts the assumption $x \notin U_{2 \epsilon}$. The theorem is proved.

Theorem 2. Every C-group is a topological group.

Proof. It is easy to see that the mapping $A \rightarrow A, x \mapsto-x$ is continuos. We have to show that the mapping $f: A \times A \rightarrow A,(x, y) \mapsto x+y$ is continuous, too. Let $U$ be an open $C$-ball of radius $\epsilon$ around $x_{0}+y_{0}$, and let $V$ and $W$ be open $C$-balls of radius $\frac{\epsilon}{2}$ around $x_{0}$ and $y_{0}$, respectively. Then $V \times W$ is an open neighbourhood around $\left(x_{0}, y_{0}\right)$. Take $(x, y)=z \in V \times W$. Then $\epsilon-N\left(x_{0}+y_{0}-(x+y)\right) \geq$ $\frac{\epsilon}{2}-N\left(x_{0}-x\right)+\frac{\epsilon}{2}-N\left(y_{0}-y\right) \in C$. Since $(x, y)$ can be chosen arbitrarily, we have $f(U \times V) \subset U$, so the continuity is proved. According to theorem $1, A$ is a Hausdorff space. Therefore, $A$ is a topological group.

\section{CONCLUDing REMARKS}

One can define an analogue of Cauchy sequence in a $C$-group $(C$-Cauchy sequence). It can be shown in a standard manner (but not so easily) that every $C$-group can be "completed". The "completion" $\hat{A}$ of a $C$-group $A$ is a $C$-group with the properties:

(i) $A$ is dense in $\hat{A}$,

(ii) $\hat{A}$ is $C$-complete (every $C$-Cauchy sequence with terms from $\hat{A}$ has limit in $\hat{A})$.

Moreover, it can be shown that $\hat{A}$ has a structure of ordered linear real space with semilinear topology. Such spaces are of special interest (see, for example [2]).

\section{REFERENCES}

1. N. Bourbaki, Algèbre II, Masson, Paris, 1981. MR 84d:00002

2. Z. Kominek and M. Kuczma, Theorems of Bernstein-Doetsch, Piccard and Mehdi and semilinear topology, Arch. Math. 52 (1989), 595-602. MR 90i:46017

University of Zagreb, Faculty of Chemical Engineering and Technology, Marulićev Trg 19, P.P. 177, 10000 Zagreb, Croatia

E-mail address: igusic@pierre.fkit.hr 